\title{
Improving surgical inpatient ward lists in a large acute hospital: a simple yet effective process to save the time of junior house officers
}

\author{
Ross Kenny, Carolyn Johnston, Imran Qureshi
}

St Georges Hospital

\begin{abstract}
In order for the smooth running of a surgical firm, an effective ward list must be created, updated, and edited each day, often by junior medical personnel. Ward lists are used by various healthcare professionals including consultants, specialist nurses, and pharmacists. Over time ward inpatient lists can become increasingly difficult to use and lacking in vital information.

Baseline measurement revealed the extent of the problem with junior house officers spending on average 95 minutes per day maintaining the ward list. After a period of research and learning, a bespoke inpatient list was created containing all of the vital information required. Criteria to fulfil included being straightforward to manipulate, easy to input new patients and aesthetically pleasing. After a trial period with modifications, an improved inpatient ward list was successfully implemented. Post-intervention data collection revealed a reduction of 42 minutes per day on average spent maintaining the list, with a $100 \%$ increase in satisfaction, and reduction in problems encountered from daily to weekly. Following this success, the general surgery weekend handover list was improved using the same prototype. This led to a saving of 8 minutes per day on average and increased doctor satisfaction.
\end{abstract}

The process of creating an effective, easy to use, and useful inpatient ward list can lead to large amount of time saved each day for the staff responsible for its management. This time can then be reinvested on clinical duties, or education, to further improve the healthcare service we provide.

\section{Problem}

St George's Healthcare is the largest healthcare provider in south west London, serving a diverse patient population of 1.3 million. A typical general surgery firm may have upwards of 50 patients split across 12 different wards throughout the hospital at any one time. Given such a high workload it is imperative to have an efficient ward list to ensure patients do not get missed.

Current issues with the current list format are (1) they are often lacking in vital information such as ward telephone numbers or emergency bleep extensions, and (2) it is very difficult to format given that it is made up of multiple tables of varying size. This can directly affect patient care firstly by missing vital patient information, and secondly by detracting junior doctors away from their clinical duties in order to manage the list. A quality improvement project was designed to create a new handover list that works seamlessly, is easily editable and can be used for future teams to be more efficient and spend their time focused on patients and not on updating lists.

If the project is successful it would be rolled out for use as the weekend general surgery handover list. At present this list is incredibly difficult to add patients to and move patients around; it's poor quality has been agreed informally amongst all teams within general surgery (Upper GI, Lower GI and Breast).
This project is focused on creating an effective inpatient ward list to be used throughout the firm including junior and senior medical staff, specialist nurses, and pharmacists. The information on these therefore needs to be displayed in a clear, concise manner. This is a vital element to the overall process of handover of information between healthcare professionals. Having an effective inpatient document that is easy to use and read forms part of the best medical practice outlined by the British Medical Association (1). Effective and safe handover of information between colleagues is also a major element in the GMC guidelines on good medical practice (2). Handover is a topic of great interest within the medical community as a good handover is the platform of continuing a high standard of care. Building an effective inpatient ward list that is easy to use is part of the process of shifting handover to an electronic domain. This has been shown in previous studies to shorten hospital stay as a result (3). Integrating information technology into clinical practice and providing tools in which to communicate knowledge more effectively has been shown to lead to substantial benefit, most notably through reduced errors and improved communication leading to a direct reduction in adverse events (4). Through this ethos the project aim is to use the best available technology to produce an effective inpatient ward list.

Following identification of the problem, formal feedback on the current list situation in the form of a questionnaire was circulated to junior medical staff within the Upper GI General surgery firm, to include any specific issues they would like to be resolved.

After searching the literature no papers were found giving guidance

\section{Background}


on the best available tools to building an effective inpatient handover list. Research was conducted through Microsoft Office regarding how to build tables that are easy to manipulate. This process involved a Microsoft Office tutorial learning module (5). The knowledge and capability to create an effective inpatient ward list to include all necessary data was acquired.

\section{Baseline measurement}

A wider perspective on the problem identified was obtained through junior medical staff feedback. Measurement for this project was carried out in the form of questionnaire feedback. This feedback contained both qualitative and quantitative measures in order to fully assess the scale of the problem identified. It also allows comparison after the intervention in order to appraise the intervention.

The questionnaire provides quantitative measurements for the following areas;

1. Time spent updating the list (pre-morning ward round and at the end of the day)

2. Ease of use of the list (scale of 1-10, 1 being very easy and 10 being very difficult)

3. Frequency of problems encountered with the current list format (scale of daily through to never)

As well as qualitative responses looking at;

1. Current satisfaction with the list (yes, no or unsure)

2. Areas to be improved upon (Open answer section)

3. Improvements for the weekend surgical handover list (to be implemented based on the success of the Upper Gl firm improvement project)

The data collected from stake-holders (house officers) will provide;

1. A comparison for the measurement of this project

2. Suggestions highlighting areas needed to be improved, which could influence the planning process of the new list to be created

3. A reflective tool following the implementation of the new list, with the subjective reviews and ideas for further improvement to be incorporated into further PDSA cycles.

From the baselines measurement, the results were as follows (evidence attached);

1. Time spent each morning updating the list: 20 minutes

2. Time spent each evening updating the list: 75 minutes

3. Total time spent daily updating the list: 95 minutes

4. How easy is it to update the list: $7 / 10$ ( 1 being very easy, 10 being very difficult)

5. How easy is it to add new patients to the list: $6 / 10$ ( 1 being very easy, 10 being very difficult)

6. On average how often do you encounter problems with the list: Daily

7. Are you satisfied with the current list format: $100 \%=$ No
8. Please outline what changes you believe need to be made to the list:

"Unification of all wards into a single table that is easy to add patients to"

"Easier to add blood results to"

1. Are you satisfied with the weekend surgical handover list: $100 \%=$ No

2. Please outline what changes you believe need to be made to the weekend handover list:

"Standardised formatting"

"Correct ordering of patients - at present they can be all over the place with different teams adding their patients ad-hoc"

Baseline measurement confirmed a shared issue with the firm handover list and that there was scope for an improvement to be made.

See supplementary file: ds3216.docx - "Baseline measurement results"

\section{Design}

The intervention itself was a straightforward process: feedback from firm members was taken on board as to how they would like the list to be designed and formatted. In order to improve upon the surgical handover list, the main problem identified through baseline measurement was that it was difficult to format. Microsoft Office, using the Word 2010 program, was chosen as the program in which the handover list would be created upon as all trust computers at St George's Hospital use Microsoft Windows operating system and have access to this program. Following research into Microsoft Word table management, one main table containing each ward known to regularly admit surgical patients was created. Dimensions were formatted so that it was easy to add new rows to the table to allow for new patients to be added to the list, or moved between wards. Colour coding of wards then took place reflecting the floor of the hospital in which the ward was situated. The order of the wards was such that it reflects the usual order of wards taken on the Upper Gl firm ward round. With each new ward, every available phone and fax number was added to the list, to hasten communication. Finally a separate table at the top of the first page was created in smaller font providing: (1) bleep/extension of every personnel member within the firm, (2) Radiology offices numbers i.e. CT, MRI, USS, etc, and (3) SpR on call bleeps for each specialty available. Taking on board criticism of the previous list that plans were not clear, a clearly defined plan column was inserted so that each patient required a plan to be in-putted. Font and font sizes for entry onto the table was standardised and fixed, so that when inputting or copying data into the list, it was entered in a standard font and size. This helps greatly to avoid formatting problems. Finally the header/footer was entered with the current date and page number respectively. 
With the template for the new list generated, patients on the existing old list were successfully copied across to the new list, which was saved in the shared file drive on the ward network for access by all members of the firm.

Note: The table was created in Microsoft office Word 2010, and also tested in Microsoft office Word 2003 to ensure compatibility across both programs, as computers throughout the trust are variable with regards to which program they have access to.

\section{Strategy}

PDSA cycle 1: Baseline measurement took place through a preintervention questionnaire within the Upper GI firm. The new Upper Gastrointestinal Surgery handover list was then based on the recommendations from stakeholder engagement. The inpatient list was then transcribed onto the prototype list. As it was a prototype it was rolled out alongside the existing handover list to retain continuity and familiarity on the ward round. The team was informed that the quality improvement project was taking place to improve the handover list, and was met with a good response as the poor quality of the existing list was well known throughout the firm. Each member of the team requiring a list $(\mathrm{F} 1, \mathrm{SHO}, \mathrm{SpR}$, Consultant and nurse specialist) had both types of list on the ward round to use. As expected this was a minor nuisance with additional paperwork to carry around. However it allowed the improvement of the new list to permeate amongst the team with numerous occasions where quick recall of an investigation, or post-operative plan was requested and the information shown to be readily and clearly available on the new list, but not on the existing one. Following three days of trialling the list and stakeholder engagement, informal and formal feedback was gained in the form of team meeting discussion and post-intervention questionnaire. The comments and feedback was taken on board for the next PDSA cycle.

PDSA cycle 2: Following the success and encouraging feedback of the prototype list, and taking on board comments from stakeholders at the firm weekly team meeting, the handover list was adapted and the final model released into circulation. The response was good, with a significant reduction in time spent maintaining/updating the list. The intervention had proven to be successful and so as a result the original list was withdrawn.

PDSA cycle 3: After the success gained with creating a new handover list for the Upper Gl firm, the same prototype was used to improve the general surgery weekend handover list; a list that all general surgery firms use (Upper $\mathrm{Gl}$, Colorectal, Breast) in order to create one 'mega-list' for patients to be seen. A repeat process of stakeholder engagement (medical staff from each general surgery firm) took place via a pre-intervention questionnaire; an adaptation from the original used in PDSA cycle 1. The new weekend general surgery handover list was created with all wards and bed numbers in columns included - allowing teams to add their patients to the correct bed so that the list was correctly ordered - a common complaint discovered from the stakeholder engagement. During a trial weekend the implementation of the new general surgery weekend handover list occurred, gaining positive reviews from other on-call junior doctors and registrar. The template for the list following its success was then made available on the shared drive, to be used for subsequent weekends. A post-intervention questionnaire was then completed with stakeholders after a period of four weekends, allowing all stakeholders to experience the new list.

See supplementary file: ds3217.docx - "PDSA Cycles in full"

\section{Post-measurement}

From the baseline measurement it was concluded that on average each house officer spends 95 minutes daily updating and managing the handover list. It was found to be difficult to both update and to add new patients to $(7 / 10$ and $6 / 10$ respectively) with problems encountered daily and $100 \%$ dissatisfaction with the list. Following intervention with the new list created, time spent on average updating the list decreased by 42 minutes to 53 minutes daily. The list was found to be easier to update/add patients to, both scoring $2 / 10$ for difficulty. Finally, problems with the list were encountered less frequently (now weekly instead of daily) with $100 \%$ satisfaction with the new list.

For the general surgery department weekend handover list (PDSA cycle 3 ), the improvement was less notable. Time spent on average updating the list for each house officer decreased by 8 minutes from 26 minutes to 18 minutes daily. Difficulty updating the list decreased from $7 / 10$ to $4 / 10$. The largest improvement was seen in the correct ordering of the list; with $100 \%$ of patients found to be in the correct order on the new list design. The general satisfaction with the weekend list remained controversial as only $62.5 \%$ of house officers reported feeling satisfied with the list after the new design was implemented.

See supplementary file: ds3228.docx - "Measurement results for each PDSA cycle"

\section{Lessons and limitations}

Undertaking a quality improvement project has been an excellent introduction into how to go about improving the working environment. By taking something small, such as a handover list, and improving it for the benefit of others, it has allowed exposure to be creative as well as make a tangible improvement to the running of the firm.

There were several limitations present whilst carrying out this project. Firstly the junior medical staff on the Upper Gl firm consist of three junior house officers and one senior house officer. Therefore when measuring the effect of the improvement it is inherently limited by the small cohort size. The small cohort size means that the study is underpowered to produce statistical significant results.

This was improved when extrapolated to the general surgery weekend handover list (fourteen junior house officers) but still this represents a small sample size when measuring data quantitatively. 


\section{BMJ Quality Improvement Reports}

The second major limitation is that given the different nature of surgical and medical firms, the list design was not ideal to be used on a medical firm, where often patients are located across a maximum of two to three wards and inpatient numbers are generally lower at St George's hospital. The new prototype list is specifically designed for patients spread out across many different wards. This provided a barrier to rolling out the intervention further.

Finally with regards to the measurement it was based on honest answering of the questionnaire. Given no competing interest it was assumed the questionnaire was answered with minimal bias, however it would have been an improved test to electronically measure online precisely how long each house officer spends editing the list. For future projects based around saving time for junior medical staff this would be a considerable improvement.

\section{Conclusion}

This was a simple yet worthwhile quality improvement project to undertake, stemming from the observation that the handover list was difficult to manage and time consuming. From carrying out questionnaire, the extent of the problem was revealed. Using creativity and research, a new list was designed. It was remarked upon as 'aesthetically pleasing' in the comments from other members of the firm, with a focused attention to small details such as colour coding and equal spacing. The time spent on updating the list was reduced from an average of 95 minutes to 53 minutes per house officer - a saving of 42 minutes per day, just by basic research into how to effectively format a list. Using the BMJ costcalculator tool this is equivalent to a cost saving of over $£ 10,000$ per annum for the department. General satisfaction with the list improved, adding to the positive culture of the firm. From spending time focusing on how to best format the list, problems encountered by house officers reduced from daily to weekly. The prototype was then replicated to improve the weekend surgical handover list; observing the same benefit as with the Upper Gl firm, albeit to a lesser degree.

\section{References}

1. British Medical Association. Safe Handover Safe Patients, Guidance on clinical handovers for patients and managers. London, http://bma.org.uk/-/media/Files/PDFs/Practical\%20advice\%2 0at\%20work/Contracts/safe\%20handover\%20s

2. General Medical Council. Good medical practice. London. (2013)

3. Ryan S, O'Riordan, JM, et al. Impact of a new electronic handover system in surgery. International Journal of Surgery $2011 ; 9(3): 217-220$

4. Bates D \& Gawande A. Improving Safety with Information Technology. The New England Journal of Medicine, ISSN 0028-4793, 06/2003, Volume 348, Issue 25, pp. 2526 - 2534

5. Microsoft office tutorials: Tables 1. Stephanie Kreiger. Accessed via: http://office.microsoft.com/en-gb/word-help/ta bles-i-create-and-format-basic-tables-RZ001200716.aspx

\section{Declaration of interests}

Nothing to declare.

\section{Acknowledgements}

Carolyn Johnston, Imran Qureshi and the general surgery house officers at St George's Hospital, London. 